\title{
Isolation and characterization of twelve microsatellite loci for the Japanese Devilray (Mobula japanica)
}

\author{
Marloes Poortvliet · Felipe Galván-Magaña • \\ Giacomo Bernardi · Donald A. Croll • \\ Jeanine L. Olsen
}

Received: 18 April 2011/Accepted: 25 April 2011/Published online: 10 May 2011

(C) The Author(s) 2011. This article is published with open access at Springerlink.com

\begin{abstract}
Twelve polymorphic microsatellites loci were characterized for Mobula japanica (Japanese Devilray) using an enrichment protocol. All but two loci were in Hardy-Weinberg equilibrium with no evidence of linkage disequilibrium or null-alleles for a sample of 40 individuals from two populations. The number of alleles varied from 5 to 28. Expected heterozygosity ranged from 0.2332 to 0.9589 , making these microsatellite loci good candidates for population genetic studies.
\end{abstract}

Keywords Elasmobranch - Mobula japanica .

Microsatellite $\cdot$ Population genetics · Polymorphism

The Japanese Devilray (Mobula japanica) is believed to have a circumglobal distribution throughout all temperate and tropical seas, although genetic analyses may identify separate populations or even cryptic species over such a wide range (Notarbartolo-di-Sciara 1987). The species reaches a disc width (DW, measured from wingtip to wingtip) of $310 \mathrm{~cm}$. It is mainly pelagic and found inshore,

\section{Poortvliet $(\bowtie) \cdot$ J. L. Olsen}

Department of Marine Benthic Ecology and Evolution,

Centre for Ecological and Evolutionary Studies,

University of Groningen, Centre for Life Sciences, Nijenborgh 7,

9747 AG Groningen, The Netherlands

e-mail: marloespoortvliet@hotmail.com

M. Poortvliet · G. Bernardi · D. A. Croll

Department of Ecology and Evolutionary Biology,

University of California Santa Cruz, 100 Shaffer Rd.,

Santa Cruz, CA 95060, USA

F. Galván-Magaña

Centro Interdisciplinario de Ciencias Marinas (CICIMAR-IPN),

Apartado postal $592 \mathrm{La} \mathrm{Paz,} \mathrm{Baja} \mathrm{California} \mathrm{Sur,} \mathrm{Mexico}$ offshore and, possibly, in oceanic environments (Last and Stevens 1994). M. japanica is listed as "Near Threatened" by the International Union for Conservation of Nature (IUCN: www.iucn.org/redlist), due to high (by) catch rates, increasing demand and low reproductive potential. Therefore, data regarding current genetic structure and migration patterns are needed to design effective conservation strategies (Graves 1998). Species-specific microsatellite markers provide a means of obtaining these data for threatened and endangered taxa. Here we report on the isolation and characterization of 12 novel microsatellite loci in M. japanica.

Genomic libraries enriched for microsatellite motifs were constructed by Genetic Identification Services (GIS, http://www.genetic-id-services.com; Chatsworth, CA, USA). Libraries were built using a sample containing $100 \mu \mathrm{g}$ of genomic DNA extracted from tail tissue of a single individual M. japanica collected in El Pardito, Baja California Sur, Mexico. The sample was stored in $90 \%$ ethanol and extracted using a Qaigen Blood and Tissue DNA purification kit. Libraries were enriched for CA, CATC, TACA, TAGA motifs. GIS sequenced 54 microsatellite-containing clones using universal M13 primers, and designed primers using DesignerPCR version 1.03 (Research Genetics, Inc.).

We tested these 54 microsatellites, using a dye-labeled universal primer system (Schuelke 2000) with an M13 tagged tail (5'-CAC GAC GTT GTA AAA CGA C-3') added to the $5^{\prime}$ end of the forward primer. Amplification reactions were carried out in a single nested reaction on an Applied Biosystems GeneAmp PCR 9700 in a total volume of $12 \mu \mathrm{L}$ containing $1 \times$ PCR Mastermix $(2.5 \mathrm{mM}$ TAPS pH9.5, $5.0 \mathrm{mM} \mathrm{KCl}, 0.2 \mathrm{mM} \mathrm{MgCl}, 20.0 \mu \mathrm{M}$ of each dNTP, Taq $0.5 \mathrm{u} / \mu \mathrm{L}$, Thermo Scientific), 2 pmols of the M13 labeled forward primer, 9 pmol of the reverse primer, 
9 pmols of the fluorescently-labeled M13 primer (Fluo, Tamra, Hex; Sigma-Genosys) and approximately 2 ng of DNA template. The following PCR temperature profile was used: $5 \mathrm{~min}$ at $94^{\circ} \mathrm{C}$, followed by 10 cycles of $30 \mathrm{~s}$ at $94^{\circ} \mathrm{C}$, $45 \mathrm{~s}$ at the primer specific $\mathrm{T}_{\mathrm{a}}, 45 \mathrm{~s}$ at $72^{\circ} \mathrm{C}$, followed by 20 cycles of $30 \mathrm{~s}$ at $94^{\circ} \mathrm{C}, 45 \mathrm{~s}$ at ( (primer specific $\mathrm{T}_{\mathrm{a}}$ ) minus $\left.2^{\circ} \mathrm{C}\right), 45 \mathrm{~s}$ at $72^{\circ} \mathrm{C}$ and a final extension of $72^{\circ} \mathrm{C}$ for $10 \mathrm{~min}$. Microsatellite amplifications were mixed with Applied Biosystems GeneScan 500 Rox size standard and then run on an ABI 3100 automated sequencer, and scored using the software GENEMAPPER3.7 (Applied Biosystems). Twelve out of the original 54 loci produced successful PCR amplification. Locus-specific dye-labeled primers (6FAM, NED, PET, VIC: Applied Biosystems) were used for those 12 loci.

Allelic diversity and heterozygosity were estimated using 40 individuals from two populations (20 from Puerto Lopez, Ecuador and 20 from La Paz, Baja California, Mexico). All 12 microsatellites were amplified in two independent multiplex reactions (Applied Biosystems GeneAmp PCR 9700; Panel 1 or 2, Table 1). The PCR reaction volume of $10 \mu \mathrm{L}$ contained $5 \mu \mathrm{L}$ Multiplex-PCR Master Mix (QIAGEN), $1 \mu \mathrm{M}$ Q-solution (QIAGEN), $2 \mu \mathrm{M}$ of each primer (Table 1 ) and $0.5 \mu \mathrm{L}$ template DNA.

Table 1 Characterization of twelve polymorphic microsatellite loci in Mobula japanica

\begin{tabular}{|c|c|c|c|c|c|c|c|c|}
\hline Locus & $\begin{array}{l}\text { Genbank } \\
\text { acc. no. }\end{array}$ & $\begin{array}{l}\text { Primer } \\
\text { label }\end{array}$ & Panel & Primer sequence $\left(5^{\prime}-3^{\prime}\right)$ & Repeat motif & $\begin{array}{l}\text { Amp. } \\
\text { range }\end{array}$ & $\mathrm{N}_{\mathrm{a}}$ & $H_{\mathrm{o}} / H_{\mathrm{e}}$ \\
\hline MOJA2 & JF800912 & $6 \mathrm{FAM}$ & 2 & $\begin{array}{l}\text { F: AGGAATGCTCCAAATAAGA } \\
\text { R:ACGTCTTCATAGCAGCAGTA }\end{array}$ & $\begin{array}{l}\text { (CA)8 TACGC (CA)4 CG (CA)5 CG } \\
\text { (CA)4 (CG)2 (CA)5 (CG)2 (CA)4 }\end{array}$ & $178-332$ & 25 & $\begin{array}{l}0.9250 \\
0.9434\end{array}$ \\
\hline MOJA4 & JF800913 & PET & 1 & $\begin{array}{l}\text { F:CAATGTCACTTTTAGCACACT } \\
\text { R:AATTCAGCGTGAGTAAACTC }\end{array}$ & (CA)3 AA (CA)30 CCT (CA)2 & $304-356$ & 28 & $\begin{array}{l}0.9750 \\
0.9589\end{array}$ \\
\hline MOJA10 & JF800914 & 6FAM & 2 & $\begin{array}{l}\text { F:GGTCTTGTTTCTGAAGTCCAGT } \\
\text { R:TGCCGATTACTAAAGAATGACA }\end{array}$ & (CA) 15 & $114-148$ & 20 & $\begin{array}{l}0.9250 \\
0.9244\end{array}$ \\
\hline MOJA112 & JF800915 & VIC & 1 & $\begin{array}{l}\text { F:CTGGCTGTTCTCTTTCCCAC } \\
\text { R:СTCCCTTCAGACCTGGACTG }\end{array}$ & $\begin{array}{l}\text { (GT)3 TTG }(\mathrm{GT}) 14 \\
\text { TTATTGTGCGTATTT (GT)3 TTA } \\
\text { (GT)4 GCTAAT (TC)2 CATTTTG } \\
\text { (GT)3 }\end{array}$ & $218-234$ & 9 & $\begin{array}{l}0.5750 \\
0.7604\end{array}$ \\
\hline MOJA124 & JF800916 & PET & 2 & $\begin{array}{l}\text { F:GCAAAAAAAGACACTGAACTGA } \\
\text { R:GACCTGAAGCATCAACTGTTTA }\end{array}$ & $(\mathrm{CA}) 10$ & $124-140$ & 8 & $\begin{array}{l}0.8000 \\
0.7756\end{array}$ \\
\hline MOJA133 & JF800917 & VIC & 2 & $\begin{array}{l}\text { F:TCCCGTAAACACTCACAGG } \\
\text { R:ATTTCTTCCCCATTCTGATG }\end{array}$ & (CA)4 TG (CA)13 & $208-226$ & 5 & $\begin{array}{l}0.7250 \\
0.6994\end{array}$ \\
\hline MOJA134 & JF800918 & VIC & 1 & $\begin{array}{l}\text { F:CCTTTACGCACACATACAAAC } \\
\text { R:CACCATCAACCCTTTCTAAGA }\end{array}$ & $\begin{array}{l}\text { (CA)3 TTCATTCAAAA }(\mathrm{CA}) 2 \\
\text { TACATA }(\mathrm{CA}) 2 \text { CGTA }(\mathrm{CA}) 2 \\
\text { GATATC }(\mathrm{CA}) 2 \\
\text { GGCATAGTCATGTATA }(\mathrm{CA}) 23\end{array}$ & $148-186$ & 20 & $\begin{array}{l}\mathbf{0 . 9 2 5 0} \\
0.9348\end{array}$ \\
\hline MOJC7 & JF800919 & PET & 1 & $\begin{array}{l}\text { F:AAGCCCTGGTGTGTGTCTG } \\
\text { R:TTTGGTAATGAAATGGAACTGG }\end{array}$ & (GTAT)4 AT (GTAT)3 & $128-156$ & 6 & $\begin{array}{l}0.2500 \\
0.2332\end{array}$ \\
\hline MOJD9 & JF800920 & $6 \mathrm{FAM}$ & 1 & $\begin{array}{l}\text { F:TGCTTTGAGACTGGTTTGC } \\
\text { R:TGGGAACTTTTACTGAGAGGG }\end{array}$ & $\begin{array}{l}\text { (CT)5 (ATCT)4 CT (ATCT)3 AC } \\
(\mathrm{CT}) 3 \text { ATCTGTCTATCTT }(\mathrm{CT}) 3 \\
\text { CCTT }(\mathrm{CT}) 2\end{array}$ & $120-144$ & 7 & $\begin{array}{l}0.4750 \\
0.4994\end{array}$ \\
\hline MOJD10 & JF800921 & VIC & 2 & $\begin{array}{l}\text { F:ACTTATTTCCATCCGGCATAGT } \\
\text { R:TCCAGGATATAAAGCGCAGTAG }\end{array}$ & (TATC)6 & $236-272$ & 8 & $\begin{array}{l}0.7000 \\
0.5972\end{array}$ \\
\hline MOJD104 & JF800922 & NED & 2 & $\begin{array}{l}\text { F:TGGCACATAATGATGATGATG } \\
\text { R:AGGATGGTAGAGGAACTCAGTG }\end{array}$ & (TAGA)9 & $256-280$ & 10 & $\begin{array}{l}\mathbf{0 . 8 5 0 0} \\
0.8548\end{array}$ \\
\hline MOJD112 & JF800923 & PET & 2 & $\begin{array}{l}\text { F:AAAATGCAGCCAGAACATG } \\
\text { R:CGCACTTGTAATGCTACTGTG }\end{array}$ & $\begin{array}{l}\text { (TAGA)7 TTGACAGA (TAGA)5 } \\
\text { CAGA (TAGA)2 (CAGA)2 } \\
\text { TAGACAGA (TAGA)2 } \\
\text { CAAATAGACAGATAGATAGG } \\
\text { (TAGA)3 TTGA (CAGA)2 } \\
\text { TAGATAAA (CAGA)2 TAGA } \\
\text { (CAGA)2 TAGACAGA (TAGA)2 } \\
\text { CAAATAGACAGATAGATAGG } \\
\text { (TAGA)2 }\end{array}$ & $148-400$ & 21 & $\begin{array}{l}0.9750 \\
0.8791\end{array}$ \\
\hline
\end{tabular}

GenBank accession numbers, primer label, amplification multiplex panel, forward (F) and reverse (R) primer sequences, repeat motif and amplification range are given for each locus. $\mathrm{N}_{\mathrm{a}}, \mathrm{H}_{\mathrm{O}}$ and $\mathrm{H}_{\mathrm{E}}$ represent number of alleles, observed heterozygosity and expected heterozygosity identified from 40 assayed individuals. Observed heterozygosity numbers in bold show significant deviations from HWE $(P<0.05)$ 
The following PCR temperature profile was used: $15 \mathrm{~min}$ at $95^{\circ} \mathrm{C}$, followed by 35 cycles of $30 \mathrm{~s}$ at $94^{\circ} \mathrm{C}, 90 \mathrm{~s}$ at $57^{\circ} \mathrm{C}, 60 \mathrm{~s}$ at $72^{\circ} \mathrm{C}$ and a final extension of $72^{\circ} \mathrm{C}$ for $10 \mathrm{~min}$. Diluted microsatellite amplifications (1:10) were mixed with Applied Biosystems GeneScan 500 LIZ size standard and then run on an ABI 3100 automated sequencer, and scored using the software GENEMAPPER3.7 (Applied Biosystems). Tests for zygotic (HardyWeinberg) equilibrium and gametic disequilibrium were conducted in Arlequin version 3.5 (Excoffier et al. 2005). A search for null alleles was conducted using Microchecker version 2.2.3 (van Oosterhout et al. 2006). We observed 5-28 alleles per locus (Table 1), with an average of 14 alleles per locus. Expected heterozygosity values ranged from 0.2332 to 0.9589 (Table 1). All loci except two (A134 and D104) were in Hardy-Weinberg equilibrium (HWE). An exact test for linkage disequilibrium between loci within the populations showed no locus pairs with significant $P$-values after Bonferroni correction. There was no evidence for null-alleles as judged empirically or from Microchecker (van Oosterhout et al. 2006).

Acknowledgments This research was funded by the Monterey Bay Aquarium and a PhD grant to MP from the University of Groningen's TopMaster-Evolutionary Biology Program. We would like to thank Devon Pearse for advice on fluorescent labeling of PCR products;
Colombo Estupiñán-Montaño for collection of samples in Ecuador; Pablo Cuevas, Felipe Cuevas and Juan Cuevas for providing help with fieldwork in Mexico; and Island Conservation for help with fieldwork. FGM thanks Instituto Politécnico Nacional (COFAA and EDI) for a fellowship.

Open Access This article is distributed under the terms of the Creative Commons Attribution Noncommercial License which permits any noncommercial use, distribution, and reproduction in any medium, provided the original author(s) and source are credited.

\section{References}

Excoffier L, Laval G, Schneider S (2005) Arlequin version 3.0: an integrated software package for population genetics data analysis. Evol Bioinform Online 1:47-50

Graves JE (1998) Molecular insights into the population structures of cosmopolitan marine fishes. J Hered 89:427-437

Last PR, Stevens JD (1994) Sharks and rays of Australia. CSIRO, Australia, p 513

Notarbartolo-di-Sciara G (1987) A revisionary study of the genus Mobula Rafinesque, 1810 (Chondrichthyes: Mobulidae) with the description of a new species. Zool J Linn Soc 91:1-91

Schuelke M (2000) An economic method for the fluorescent labeling of PCR fragments. Nature 18:233-234

van Oosterhout CD, Weetman D, Hutchinson WF (2006) Estimation and adjustment of microsatellite null alleles in non-equilibrium populations. Mol Ecol Resour 6:255-256 